\title{
Morbidity pattern among geriatric population in urban field practice area of district of Maharashtra: a cross sectional study
}

\author{
Swapnil Jain $^{1 *}$, Alka C. Kaware ${ }^{2}$, Mohan K. Doibale ${ }^{3}$, Shabana Shaikh ${ }^{4}$, Radhey BK ${ }^{1}$, \\ Madhuri P. Mathurkar ${ }^{1}$
}

${ }^{1}$ Department of Community Medicine, Government Medical College, Miraj, Maharashtra, India

${ }^{2}$ Department of Community Medicine, Indira Gandhi Government Medical College, Nagpur Maharashtra, India

${ }^{3}$ Department of Community Medicine, Government Medical College, Aurangabad, Maharashtra, India

${ }^{4}$ Department of Community Medicine, Topiwala National Medical College and BYL Nair Hospital Mumbai Central Maharashtra, India

Received: 16 December 2015

Accepted: 08 January 2016

\section{*Correspondence:}

Dr. Swapnil Jain,

E-mail: sjvicky85@gmail.com

Copyright: (C) the author(s), publisher and licensee Medip Academy. This is an open-access article distributed under the terms of the Creative Commons Attribution Non-Commercial License, which permits unrestricted non-commercial use, distribution, and reproduction in any medium, provided the original work is properly cited.

\begin{abstract}
Background: There is a rapid expansion of geriatric age group due to increased longevity \& so the morbidities among them, Morbidity pattern in geriatric age group need to be studied extensively for formulating effective strategies to improve their health status. The objectives of the study were to assess some specified morbidities among geriatric population and to study association of socio-demographic factors with morbidities among geriatric population.

Methods: A Community based cross sectional study was conducted in the urban slums, field practice area of Community Medicine attached to a tertiary care hospital. Study period was Jan 2013-Dec 2014 .Persons aged $\geq 60$ years residing in the urban slums were included. Total 600 elderly persons were studied. A pre-designed, pre-tested semi structured questionnaire was used to collect information. Data was entered in Microsoft excel and analysed by using frequency, proportion, chi-square test with SPSS 16.

Results: Out of 600 subjects, majority $65.3 \%$ had hearing impairment, followed by anemia $62.2 \% \mathrm{HTN}, 46.2 \%$, joint pain $42.3 \%$, dental problems $41.0 \%$, cataract 38.7\%, chronic bronchitis $31.8 \%$, APD 27.3\%, DM 11\%, skin diseases $7.5 \%$, piles $7.2 \%$, BPH $3.7 \%$ and TB 1.0\%. Various socio-demographic variables were found to be significantly associated with morbidities. Majority $486(81 \%)$ had multiple morbidity, $89(14.8 \%)$ had single and only $25(4.2 \%)$ were not suffering from any type of morbidity.

Conclusions: The study presented higher rate of Morbidity among elderly and its interesting association with various socio-demographic variables which may have important implications in planning for various health care delivery services for elderly.
\end{abstract}

Keywords: Elderly, Urban, Geriatric, Morbidity, Hypertension

\section{INTRODUCTION}

The problems of the aged differ not only between nations but also within nations and between groups. ${ }^{1}$ Older people are considered as a special group for following reasons: Icebergs of disease, Multiple pathology, Loss of adaptability, Costs of care. ${ }^{2}$ According to world health statistics 2014, globally around $11 \%$ of population is above 60 yrs of age, and $8 \%$ of population is above 60 yrs of age in South East Asian countries including India. ${ }^{3}$ 
Between 2000 and 2050, the proportion of the world's population over 60 years will double from about $11 \%$ to $22 \%$. The number of people aged 60 years and over is expected to increase from 605 million to 2 billion over the same period 4Considering the increasing burden of geriatric health and social problems in India, the World Health Organization (WHO) in collaboration with the Government of India carried out a cross-sectional, community based study of the elderly population 60 years and above at 10 different sites in different states and union territories of India. ${ }^{5}$ The Indian Council of Medical Research estimated the magnitude of common illnesses among older people for the projected population of $1996 .^{6}$

So due to vast number of morbidities in elderly people and lack of adequate health care system to tackle the issues, a thorough examination of the morbidity profiles among the elderly and an assessment of the related socio demographic factors are required to improve the delivery of health care to the elderly. In view of above context this study was conducted to assess some specified morbidities and to study association of socio-demographic factors with morbidities among geriatric population in a district of Maharashtra.

\section{METHODS}

The present study was carried out at the Urban field practice area and was part of thesis conducted under department of Community Medicine attached to tertiary care hospital .Study period was Jan 2013 to Dec 2014. Ethical approval was taken from Institutional Ethical Committee. It was Community based cross-sectional study. Total 600 elderly subjects were studied. The total 11 wards under field practice area of urban health centre of a Medical College in a city of Maharashtra were enlisted. As wards-sampling units vary considerably in population size, probability proportional to size (PPS) sampling method was used, Study population of the age group 60 years and above enumerated for each ward by using voter (Electoral) list of 2012. ${ }^{7}$ Data was collected by semi-structured questionnaire. Data entry and statistical analyses used SPSS version 16. Frequency distributions were calculated for all variables. The chisquare test was used to test significance of associations between independent variables and morbidities with the threshold for significance set at $\mathrm{p}=0.05$.Operation definition of Morbidities were: a) Hypertension: A person was considered to be hypertensive if he/she was an already diagnosed case of hypertension and/or on treatment (known case of hypertension) or with a current systolic blood pressure $\geq 140 \mathrm{~mm}$ of $\mathrm{Hg}$ and/or diastolic blood pressure $\geq 90 \mathrm{~mm}$ of $\mathrm{Hg}^{8-9}$ b) Anemia: Already known case of anaemia and newly diagnosed case of anaemia were taken into consideration. A patient was diagnosed as anaemic on basis of hemoglobin levels done by Sahli's hemoglobin estimation method. ${ }^{10}$ Cut off for diagnosis of anaemia was decided according to WHO classification. ${ }^{11}$ c) Diabetes mellitus: Results of OGTT (2hour post OGTT plasma glucose) were interpreted as per the WHO standards. A 2-hour post OGTT plasma glucose $\geq 200 \mathrm{mg} / \mathrm{dl}$ was diagnosed as diabetes mellitus and rest were labelled as non-diabetic. ${ }^{12}$

Following morbidities in the study subjects were diagnosed in accordance by using documents and investigation reports already available with the participants, chronic bronchitis, joint pain, piles, Benign Prostatic Hypertrophy (BPH), Acid Peptic Disease (APD), cataract, hearing impairment, skin diseases and dental problems and Tuberculosis (TB).Newly diagnosed cases of DM, HTN and anaemia, were referred to a Medical College for further investigations and management.

\section{RESULTS}

Majority $65.3 \%$ had hearing impairment, followed by anaemia $62.2 \%$, HTN, $46.2 \%$, joint pain $42.3 \%$, dental problems $41.0 \%$, cataract $38.7 \%$, chronic bronchitis $31.8 \%$, APD $27.3 \%$, DM 11\%, skin diseases $7.5 \%$, piles $7.2 \%, \mathrm{BPH} 3.7 \%$ and TB $1.0 \%$. HTN, BPH, cataract and hearing impairment were significantly associated with sex of study subjects $(p<0.05)$. Anemia, DM, chronic bronchitis, joint pain, piles, APD, skin diseases, TB and dental problems were not significantly associated with sex of study subjects ( $>0.05)$ [Table 1]. All the morbidities were not significantly associated with religion $(\mathrm{p}>0.05)$.

HTN, anaemia, DM, chronic bronchitis, cataract, hearing impairment, and dental problems were significantly associated with age of study subjects. Joint pain, BPH, piles, APD, TB and skin diseases were not significantly associated with age of study subjects $(p>0.05)$ [Table 2].

APD, Skin diseases, and dental problems were significantly associated with occupation of study subjects $(\mathrm{p}<0.05)$. HTN, anaemia, DM, chronic bronchitis, joint pain, $\mathrm{BPH}$, piles, cataract, hearing impairment, TB were not significantly associated with occupation of study subjects $(\mathrm{p}>0.05)$ [Table 3].

Anemia, DM, chronic bronchitis and BPH were significantly associated with type of family $(\mathrm{p}<0.05)$. HTN, joint pain, piles, APD, cataract, hearing impairment, skin diseases, TB and dental problems were not significantly associated with type of family $(p>0.05)$ [Table 4].

DM, chronic bronchitis, piles, skin diseases were significantly associated with socioeconomic status ( $\mathrm{p}<0.05)$. HTN, anaemia, joint pain ,BPH, APD, cataract, hearing impairment, TB and dental problems were not significantly associated with socio economic status $(\mathrm{p}>0.05)$ [Table 5].

Anemia was significantly associated with educational status $(\mathrm{p}<0.05)$. HTN, DM, chronic bronchitis, joint pain, $\mathrm{BPH}$, piles, APD, cataract, hearing impairment, skin 
diseases, TB and dental problems were not significantly associated with educational status $(\mathrm{p}>0.05)$ [Table 6].
Out of total 600 subjects, majority $486(81 \%)$ had multiple morbidity, $89(14.8 \%)$ had single and only 25 $(4.2 \%)$ were not suffering from any type of morbidity.

Table 1: Distribution of morbidities among study subjects and their association with sex.

\begin{tabular}{|llllll|}
\hline Morbidity & Male & Female & Total & $\chi^{2}$ & p value \\
\hline HTN & $133(48.0)$ & $144(52.0)$ & $277(46.2)$ & 3.954 & 0.047 \\
\hline Anemia & $172(46.1)$ & $201(53.9)$ & $373(62.2)$ & 2.398 & 0.122 \\
\hline DM & $36(54.5)$ & $30(45.5)$ & $66(11.0)$ & 3.568 & 0.06 \\
\hline Chronic Bronchitis & $83(43.5)$ & $108(56.5)$ & $191(31.8)$ & 0.005 & 0.943 \\
\hline Joint pain & $120(47.2)$ & $134(52.8)$ & $254(42.3)$ & 2.292 & 0.130 \\
\hline BPH & $22(100)$ & 0 & $22(3.7)$ & 29.462 & $<0.0001$ \\
\hline Piles & $24(55.8)$ & $19(44.2)$ & $43(7.2)$ & 2.778 & 0.096 \\
\hline APD & $77(47)$ & $87(53)$ & $164(27.3)$ & 0.990 & 0.320 \\
\hline Cataract & $116(50)$ & $116(50)$ & $232(38.7)$ & 6.168 & 0.013 \\
\hline Hearing impairment & $197(50.3)$ & $195(49.7)$ & $392(65.3)$ & 19.954 & $<0.0001$ \\
\hline Skin diseases & $24(53.3)$ & $21(46.7)$ & $45(7.5)$ & 1.848 & 0.174 \\
\hline TB & $4(66.7)$ & $2(33.3)$ & $6(1.0)$ & 1.303 & 0.254 \\
\hline Dental problems & $105(42.7)$ & $141(57.3)$ & $246(41)$ & 0.164 & 0.685 \\
\hline
\end{tabular}

Figures in parenthesis denote percentages

Table 2: Association of morbidities among study subjects with age group.

\begin{tabular}{|c|c|c|c|c|c|c|c|}
\hline \multirow{2}{*}{ Morbidity } & \multicolumn{5}{|c|}{ Age(in years) } & \multirow{2}{*}{ Total } & \multirow{2}{*}{$\chi^{2}, \mathbf{p}$ value } \\
\hline & $60-64$ & $65-69$ & $70-74$ & $75-79$ & $\geq 80$ & & \\
\hline HTN & $106(38.3)$ & $67(24.2)$ & $28(10.1)$ & $39(14.1)$ & $37(13.3)$ & $277(46.2)$ & $\begin{array}{l}\chi^{2}=64.52 \\
p<0.0001\end{array}$ \\
\hline Anemia & 144(38.6) & $97(26.0)$ & $62(16.6)$ & $26(7.0)$ & $44(11.8)$ & $373(62.2)$ & $\begin{array}{l}\chi^{2}=10.53 \\
p=0.0324\end{array}$ \\
\hline $\mathrm{DM}$ & $10(15.2)$ & $25(37.9)$ & $19(28.8)$ & $06(9.1)$ & $06(9.1)$ & $66(11)$ & $\begin{array}{l}\chi^{2}=21.26 \\
p=0.0003\end{array}$ \\
\hline Chronic bronchitis & $51(26.7)$ & $50(26.2)$ & $62(32.5)$ & $21(11.0)$ & $7(3.7)$ & 191(31.9) & $\begin{array}{l}\chi^{2}=23.36 \\
p=0.0001\end{array}$ \\
\hline Joint pain & $96(37.8)$ & $59(23.2)$ & $53(20.9)$ & $20(7.9)$ & $26(10.2)$ & $254(42.33)$ & $\begin{array}{l}\chi^{2}=2.566 \\
p=0.6328\end{array}$ \\
\hline $\mathrm{BPH}$ & $9(41)$ & $3(13.7)$ & $6(27.3)$ & $2(9.0)$ & $2(9.0)$ & $22(3.7)$ & $\begin{array}{l}\chi^{2}=2.086 \\
p=0.720\end{array}$ \\
\hline Piles & $11(25.6)$ & $11(25.6)$ & $13(30.2)$ & $5(11.6)$ & $3(7.0)$ & $43(7.16)$ & $\begin{array}{l}\chi^{2}=7.233 \\
\mathrm{p}=0.124\end{array}$ \\
\hline APD & $56(34.1)$ & $39(23.8)$ & $39(23.8)$ & $15(9.1)$ & $15(9.1)$ & $164(27.3)$ & $\begin{array}{l}\chi^{2}=6.26 \\
p=0.1806\end{array}$ \\
\hline Cataract & $75(32.3)$ & $48(32.7)$ & $58(25.0)$ & $20(8.6)$ & $31(13.4)$ & $232(38.2)$ & $\begin{array}{l}\chi^{2}=23.360 \\
p=0.0001\end{array}$ \\
\hline $\begin{array}{l}\text { Hearing } \\
\text { impairment }\end{array}$ & $196(50.0)$ & $71(18.1)$ & $69(17.6)$ & $29(7.4)$ & 27(6.9) & $392(65.3)$ & $\begin{array}{l}\chi^{2}=54.294 \\
p<0.0001\end{array}$ \\
\hline Skin diseases & $19(42.2)$ & $6(13.3)$ & $7(15.6)$ & $6(13.3)$ & $7(15.6)$ & $45(7.5)$ & $\begin{array}{l}\chi^{2}=7.404 \\
p=0.1160\end{array}$ \\
\hline TB & $2(33.3)$ & $2(33.3)$ & 0 & $1(16.7)$ & 1(16.7) & $6(1.0)$ & $\begin{array}{l}\chi^{2}=2.517 \\
p=0.642\end{array}$ \\
\hline Dental problems & $78(31.7)$ & $50(20.3)$ & $42(17.1)$ & $30(12.2)$ & $46(18.7)$ & $246(41.0)$ & $\begin{array}{l}\chi^{2}=64.978 \\
p<0.0001\end{array}$ \\
\hline
\end{tabular}

Figures in parenthesis denote percentages 
Morbidity load was higher in females (97.6\%) as compared to males $(93.5 \%)$ and the difference was statistically significant $(\mathrm{P}<0.05)$ [Table 7].

Table 3: Association of morbidities among study subjects with occupation.

\begin{tabular}{|llllllll|}
\hline Morbidity & Unemployed & Unskilled & $\begin{array}{l}\text { Semi } \\
\text { skilled }\end{array}$ & Skilled & Professionals & Total & $\chi^{2}$,p value \\
\hline HTN & $193(69.7)$ & $45(16.2)$ & $21(7.6)$ & $5(1.8)$ & $13(4.7)$ & $277(46.2)$ & $7.658,0.105$ \\
\hline Anemia & $270(72.4)$ & $63(16.9)$ & $14(3.8)$ & $7(1.9)$ & $19(5.1)$ & $373(62.2)$ & $4.72,0.317$ \\
\hline DM & $49(74.2)$ & $15(22.7)$ & $1(1.5)$ & 0 & $1(1.5)$ & $66(11)$ & $5.976,0.201$ \\
\hline Chronic bronchitis & $145(75.9)$ & $27(14.1)$ & $10(5.2)$ & $3(1.6)$ & $6(3.1)$ & $191(31.8)$ & $3.685,0.450$ \\
\hline Joint pain & $194(74.6)$ & $38(14.6)$ & $9(3.5)$ & $7(2.7)$ & $12(4.6)$ & $260(42.3)$ & $6.004,0.199$ \\
\hline BPH & $13(59.1)$ & $6(27.3)$ & $1(4.5)$ & $0(0)$ & $2(9.1)$ & $22(3.7)$ & $3.44,0.487$ \\
\hline Piles & $31(72.1)$ & $8(18.6)$ & $4(9.3)$ & $0(0)$ & $0(0)$ & $43(7.2)$ & $4.695,0.320$ \\
\hline APD & $121(73.8)$ & $26(15.9)$ & $3(1.8)$ & $7(4.3)$ & $7(4.3)$ & $164(27.3)$ & $10.82,0.029$ \\
\hline Cataract & $162(69.8)$ & $44(19.0)$ & $10(4.3)$ & $4(1.7)$ & $12(5.2)$ & $232(38.7)$ & $1.82,0.769$ \\
\hline Hearing impairment & $269(68.6)$ & $74(18.9)$ & $22(5.6)$ & $9(2.3)$ & $18(4.6)$ & $392(65.3)$ & $4.238,0.375$ \\
\hline Skin diseases & $38(84.4)$ & $3(6.7)$ & $1(2.2)$ & $3(6.7)$ & $0(0)$ & $45(7.5)$ & $12.52,0.014$ \\
\hline TB & $4(66.7)$ & $2(33.3)$ & 0 & 0 & 0 & $6(1.0)$ & $1.6,0.809$ \\
\hline Dental problems & $183(74.4)$ & $37(15.0)$ & $17(6.9)$ & $3(1.2)$ & $6(2.4)$ & $246(41)$ & $9.577,0.048$ \\
\hline
\end{tabular}

Figures in parenthesis denote percentages

Table 4: Association of morbidities among study subjects with type of family.

\begin{tabular}{|lllllll|}
\hline Morbidity & Nuclear & Joint & Three Generation & Total & $\chi^{2}$ & p value \\
\hline HTN & $84(30.3)$ & $60(21.7)$ & $133(48.0)$ & $277(46.2)$ & 1.847 & 0.397 \\
\hline Anemia & $97(26.0)$ & $101(27.1)$ & $175(46.9)$ & $373(62.2)$ & 6.193 & 0.04 \\
\hline DM & $18(27.3)$ & $24(36.4)$ & $24(36.4)$ & $66(11.0)$ & 6.413 & 0.041 \\
\hline Chronic Bronchitis & $47(24.6)$ & $58(30.4)$ & $86(45.0)$ & $191(31.8)$ & 6.424 & 0.040 \\
\hline Joint pain & $69(26.5)$ & $74(28.5)$ & $117(45.0)$ & $260(43.3)$ & 4.731 & 0.094 \\
\hline BPH & $6(27.3)$ & $11(50.0)$ & $5(22.7)$ & $22(3.7)$ & 9.189 & 0.010 \\
\hline Piles & $13(30.2)$ & $13(30.2)$ & $17(39.5)$ & $43(7.2)$ & 1.286 & 0.526 \\
\hline APD & $41(25.0)$ & $46(28.0)$ & $77(47.0)$ & $164(27.3)$ & 2.558 & 0.278 \\
\hline Cataract & $65(28.0)$ & $67(28.9)$ & $100(43.1)$ & $232(38.7)$ & 4.789 & 0.091 \\
\hline Hearing impairment & $116(29.6)$ & $104(26.5)$ & $172(43.9)$ & $392(65.3)$ & 5.186 & 0.075 \\
\hline Skin diseases & $12(26.7)$ & $17(37.8)$ & $16(35.6)$ & $45(7.5)$ & 5.164 & 0.076 \\
\hline TB & $2(33.3)$ & $2(33.3)$ & $2(33.3)$ & $6(1.0)$ & 0.494 & 0.781 \\
\hline Dental problems & $76(30.9)$ & $60(24.4)$ & $110(44.7)$ & $246(41.0)$ & 1.086 & 0.581 \\
\hline
\end{tabular}

Figures in parenthesis denote percentages

\section{DISCUSSION}

In the present study, out of 600 subjects majority $65.3 \%$ had hearing impairment, followed by anaemia (62.2\%), HTN (46.2\%), joint pain (42.3\%), dental problems $(41.0 \%)$, cataract $(38.7 \%)$, chronic bronchitis $(31.9 \%)$, APD (27.3\%), DM (11\%), skin diseases (7.5\%), piles (7.2\%), BPH (3.7\%), TB (1.0\%). Percentages of elderly with anaemia were high in elderly because it is multi factorial with etiology as nutritional, physiological and pathological problems. Dental problems are a common accompaniment of ageing and in India, a largely unmet one. Poor oral Hygiene was responsible for large fraction of dental problems. There was wide variation in distribution of morbidities among geriatric persons in various studies conducted in different parts of India Qadri $\mathrm{S}$ et al found that majority were anaemic $(64.5 \%)$ and had dental problems $(62.2 \%)$, followed by joint pains $(51.4 \%)$, cataract $(46.8 \%)$, hypertension $(44.5 \%)$ respectively.

Further $25.4 \%$ were having senile deafness, $22.2 \%$ suffered from acid peptic disease, and $9 \%$ were diagnosed cases of diabetes mellitus. ${ }^{13}$ 
Kumar $\mathrm{R}$ found that prevalence of various morbidities being hypertension $(13.12 \%)$, arthritis $(11.25 \%)$, cataract $(11.87 \%)$, dental problems $(4.37 \%)$, diabetes mellitus
$(3.75 \%)$, skin problems $(3.12 \%)$, gastric trouble $(1.25 \%)$ and piles $(0 \%){ }^{14}$

Table 5: Association of morbidities among study subjects with socioeconomic status.

\begin{tabular}{|c|c|c|c|c|c|c|c|c|}
\hline \multicolumn{7}{|c|}{ Socioeconomic status } & \multirow[b]{2}{*}{$\chi^{2}$} & \multirow{2}{*}{ p value } \\
\hline Morbidity & I & II & III & IV & V & Total & & \\
\hline HTN & $3(1.1)$ & $18(6.5)$ & $40(14.4)$ & $86(31.0)$ & $130(46.9)$ & $277(46.2)$ & 0.585 & 0.965 \\
\hline Anemia & $5(1.3)$ & $26(7.0)$ & $50(13.4)$ & 114(30.6) & $178(47.7)$ & $373(62.2)$ & 5.918 & 0.205 \\
\hline DM & $1(1.5)$ & $3(4.5)$ & $4(6.1)$ & $15(22.7)$ & $43(65.2)$ & $66(11.0)$ & 11.51 & 0.021 \\
\hline Chronic bronchitis & $3(1.6)$ & $12(6.3)$ & $18(9.4)$ & $71(37.2)$ & $87(45.5)$ & 191(31.8) & 11.21 & 0.024 \\
\hline Joint pain & $3(1.2)$ & $16(6.3)$ & $30(11.8)$ & $76(29.9)$ & $129(50.8)$ & $254(42.3)$ & 5.148 & 0.272 \\
\hline BPH & $0(0)$ & $1(4.5)$ & $4(18.2)$ & $9(40.9)$ & $8(36.4)$ & $22(3.7)$ & 1.715 & 0.788 \\
\hline Piles & $1(2.3)$ & $2(4.7)$ & $4(9.3)$ & $7(16.3)$ & $29(67.4)$ & $43(7.2)$ & 9.769 & 0.045 \\
\hline APD & $3(1.8)$ & $11(6.7)$ & $20(12.2)$ & $47(28.7)$ & $83(50.6)$ & $164(27.3)$ & 5.005 & 0.287 \\
\hline Cataract & $3(1.3)$ & $15(6.5)$ & $26(11.2)$ & $67(28.9)$ & $121(52.2)$ & $232(38.7)$ & 7.33 & 0.119 \\
\hline Hearing impairment & $4(1.0)$ & $25(6.4)$ & $53(13.5)$ & $124(31.6)$ & $186(47.4)$ & $392(65.3)$ & 2.40 & 0.662 \\
\hline Skin diseases & $1(2.2)$ & $4(8.9)$ & $4(8.9)$ & $7(15.6)$ & $29(64.4)$ & $45(7.5)$ & 9.815 & 0.044 \\
\hline TB & $0(0)$ & $0(0)$ & $1(16.7)$ & $3(50.0)$ & $2(33.3)$ & $6(1.0)$ & 1.38 & 0.847 \\
\hline Dental Problems & $3(1.2)$ & $12(32.4)$ & $40(16.3)$ & $88(35.8)$ & $103(36.5)$ & $246(41.0)$ & 7.70 & 0.103 \\
\hline
\end{tabular}

Figures in parenthesis denote percentages

Table 6: Association of morbidities among study subjects with educational status.

\begin{tabular}{|c|c|c|c|c|c|c|c|c|}
\hline & \multicolumn{6}{|c|}{ Educational status } & \multirow[b]{2}{*}{ Total } & \multirow[b]{2}{*}{$P$ value } \\
\hline Morbidity & Illiterate & Primary & Middle & Secondary & $\begin{array}{l}\text { Higher } \\
\text { secondary }\end{array}$ & $\begin{array}{l}\text { Graduate } \\
\text { and above }\end{array}$ & & \\
\hline HTN & 143(51.6) & $55(19.9)$ & $32(11.6)$ & $22(7.9)$ & $16(5.8)$ & $9(3.2)$ & $277(46.2)$ & 0.16 \\
\hline Anemia & $209(56.0)$ & $67(18.0)$ & $41(11.0)$ & $24(6.4)$ & 21(5.6) & $11(2.9)$ & $373(62.2)$ & 0.03 \\
\hline DM & $40(60.6)$ & $12(18.2)$ & $4(6.1)$ & $5(7.6)$ & $2(3.0)$ & $3(4.5)$ & $66(11)$ & 0.3 \\
\hline $\begin{array}{l}\text { Chronic } \\
\text { Bronchitis }\end{array}$ & $108(56.5)$ & $35(18.3)$ & $20(10.5)$ & $15(7.9)$ & $11(5.8)$ & $2(1.0)$ & 191(31.8) & 0.91 \\
\hline Joint pain & $142(55.9)$ & 48(18.9) & $27(10.6)$ & $16(6.3)$ & $12(6.3)$ & $9(3.5)$ & 254(42.3) & 0.08 \\
\hline BPH & $13(59.1)$ & $4(18.2)$ & $3(13.6)$ & $1(4.5)$ & $1(4.5)$ & $0(0)$ & $22(3.7)$ & 0.9 \\
\hline Piles & $24(55.8)$ & $9(20.9)$ & $2(4.7)$ & $4(9.3)$ & $2(4.7)$ & $2(4.7)$ & $43(7.2)$ & 0.53 \\
\hline APD & $85(51.8)$ & $34(20.7)$ & 17(10.4) & $11(6.7)$ & $10(6.1)$ & $7(4.3)$ & 164(27.3) & 0.08 \\
\hline Cataract & $124(53.4)$ & $47(20.3)$ & $26(11.2)$ & $16(6.9)$ & $11(4.7)$ & $8(3.4)$ & $232(38.2)$ & 0.19 \\
\hline $\begin{array}{l}\text { Hearing } \\
\text { impairment }\end{array}$ & $218(55.6)$ & $74(18.9)$ & $45(11.5)$ & $26(6.6)$ & $18(4.6)$ & $11(2.8)$ & $392(65.3)$ & 0.0 \\
\hline $\begin{array}{l}\text { Skin } \\
\text { diseases }\end{array}$ & $28(62.2)$ & $7(15.6)$ & $3(6.7)$ & $3(6.7)$ & $2(4.4)$ & $2(4.4)$ & $45(7.5)$ & 0.6 \\
\hline TB & $6(100)$ & 0 & 0 & 0 & 0 & 0 & $6(1.0)$ & 0.42 \\
\hline $\begin{array}{l}\text { Dental } \\
\text { Problems }\end{array}$ & $143(58.1)$ & $39(15.9)$ & $28(11.4)$ & $16(6.5)$ & $16(6.5)$ & $4(1.6)$ & $246(41)$ & 0.37 \\
\hline
\end{tabular}

Figures in parenthesis denote percentages

In the present study, HTN, BPH, cataract and hearing impairment were significantly associated with sex of study subjects $(\mathrm{p}<0.05)$ HTN was found to be significantly more common in females in our study. Similar findings were obtained by, Bharati DR et al and Afridi $\mathrm{N}$ et al. ${ }^{15,16}$ Cataract was found to be significantly associated with sex in study conducted by Barman SK et al. ${ }^{17}$ In our study, HTN, anaemia, DM, chronic bronchitis, cataract, hearing impairment, and dental problems were significantly associated with age of study subjects ( $\mathrm{p}<0.05)$.Similarly, HTN was significantly associated with age in studies conducted by Bharati DR et al and Shraddha K et al. ${ }^{15,18}$ Anemia was significantly associated with age in studies conducted by Qadri $\mathrm{S}$ et al. ${ }^{13}$ 
Shraddha $\mathrm{K}$ et al found that hearing impairment was significantly associated with age and significant association was found between senile deafness and age in study conducted by Qadri S et al (2013). ${ }^{13,18}$ Cataract was significantly associated with age in studies conducted by Qadri S et al and Kakkar R et al. ${ }^{13,19}$

\section{Table 7: Distribution of study subjects according to number of morbidities.}

\begin{tabular}{|llll|}
\hline $\begin{array}{l}\text { No. of } \\
\text { morbidity } \\
\text { per elderly }\end{array}$ & Male & Female & Total \\
\hline No morbidity & $17(6.5)$ & $8(2.4)$ & $25(4.2)$ \\
\hline Single & $40(15.3)$ & $49(14.5)$ & $89(14.8)$ \\
\hline Multiple & $205(78.2)$ & $281(83.1)$ & $486(81.0)$ \\
\hline Total & $262(100)$ & $338(100)$ & $600(100)$ \\
\hline
\end{tabular}

Figures in parenthesis denote percentages

$\chi^{2}=6.5 ; \mathrm{df}=2 ; \mathrm{p}=0.0001$

Joint pain, dental problems and hearing impairment were significantly associated with marital status $(\mathrm{p}<0.05)$. Joshi $\mathrm{K}$ et al concluded that based on marital status higher morbidity was seen in unmarried and divorced subjects using univariate analysis with morbidity $(\mathrm{p}<0.001) .{ }^{20}$ In the present study, skin diseases, APD and dental problems were significantly associated with occupation of study subjects $(\mathrm{p}<0.05)$. Similarly, Joshi K et al found that higher morbidity was seen among workers (skilled and unskilled), those unable to work, and those involved in household chores $(\mathrm{p}<0.001)$ In present study, anaemia, DM, chronic bronchitis and BPH were significantly associated with type of family $(\mathrm{p}<0.05) .{ }^{20} \mathrm{In}$ contrast, Bharati DR et al showed that there was no significant association between type of family and morbidities like diabetes and anaemia $(\mathrm{p}>0.05) .{ }^{15}$

Anemia was significantly associated with educational status $(\mathrm{p}<0.05)$. HTN, DM ,chronic bronchitis, joint pain, $\mathrm{BPH}$, piles, APD, cataract, hearing impairment ,skin diseases, TB and dental problems were not significantly associated with educational status $(\mathrm{p}>0.05)$. Kumar $\mathrm{R}$ et al in their study found no significant association between morbidity and educational status. ${ }^{21}$ (P>0.05) Barman SK et al observed that arthritis, anaemia, hypertension, loss of teeth $\&$ dental caries were significant in relation with educational status. $^{17}$

In present study DM, chronic bronchitis, piles, skin diseases were significantly associated with socioeconomic status $(\mathrm{p}<0.05)$. Similarly, Joshi K et al concluded that lower family income was associated with higher morbidity and Bharati DR et al showed that significant association exist between diabetes mellitus and per capita income. ${ }^{15,20}$ Diabetes was more common in non BPL than in BPL. In present study, majority $(81 \%)$ had multiple morbidity, $14.8 \%$ had single and only $4.2 \%$ were not suffering from any type of morbidity. Singh JP et al revealed that majority, $98.75 \%$ had multiple morbidities and $1.25 \%$ had single morbidity. ${ }^{22}$ In contrast, Srivastava K et al found that majority $57.8 \%$ had multiple morbidities and $31.4 \%$ had single morbidity. $^{23}$

\section{CONCLUSION}

The care of elderly is drawing more and more attention of the Government and public. It is already a major social and health problem in affluent countries. The study provides data to plan services and programmes for betterment of aged hope this study yields valuable information required for the design of the service to be provided for this special group.

\section{Recommendations}

Geriatric care should become an integral part of the primary health care delivery system. Regular screening programmes for the detecting chronic diseases at the earliest should be carried out. The pattern of geriatric health problems have different characteristics and hence require a specific type of screening programme based on the finding of various geriatric health studies including the current one.

\section{Funding: No funding sources \\ Conflict of interest: None declared}

Ethical approval: The study was approved by the Institutional Ethics Committee

\section{REFERENCES}

1. Gurav RB, Kartikeyan S. Problems of geriatric population in an urban area. Bombay Hosp J. 2002;44(1).

2. Detels R, McEwen J, Beagle hole R, Tanaka H. Oxford Textbook of Public Health, 4th ed .Oxford University. 2002:1196.

3. WHO. World Health Statistics 2014.Available at http://apps.who.int/iris/bitstream/10665/112738/1/97 89240692671_eng.pdf.

4. Facts on ageing and life course. WHO ageing and life course. Available from: http://www.who.int/features/factfiles/ageing/ageing facts/en/index.html. Accessed online on November 2014.

5. World Health Organization Collaborative Programme supported by the Government of India. Multicentric study to establish epidemiological data on health problems in elderly. Available at http://www.whoindia.org/LinkFiles/Health_Care_for _the_Elderly_Multicentric_study_healthcareelderly_ exe.pdf. Last cited on 2014.

6. Dey AB. Handbook on Geriatrics.Paras Medical Publishers, Hyderabad, India. 1-93.

7. Steps in applying Probability Proportional to Size (PPS) and calculating.Basic Probability Weights [Online]. [cited on 18 Jan 2013]; Available from URL:http://www.who.int/tb/advisory_bodies/impact 
_measurement_taskforce/meetings/prevalence_surve y/psws_probability_prop_size_bierrenbach.pdf

8. Report of WHO consultation. Hypertension control. Geneva, Switzerland: WHO; TRS - 862, 1996.

9. Chobanian AV, Bakris GL, Black HR, Cushman WC, Green LA, Izzo JL Jr, et al. The seventh report of the joint national committee on prevention, detection, evaluation and treatment of high blood pressure: The JNC 7 Report. JAMA 2003; 289:256072.

10. Sanyal S Clinical Pathology: Prep manual for undergraduates. Elsevier India private limited (New Delhi). 2005:25.

11. Report of WHO consultation. Nutritional Anaemias Geneva, Switzerland: World Health Organization; TRS - 405,1968.

12. Report of a WHO consultation. Definition, diagnosis and classification of diabetes mellitus and its complications; Part 1:Diagnosis and classification of diabetes mellitus.Geneva, Switzerland: World Health Organization, 1999.

13. Qadri S ,Ahluwalia SK, Ganai AM, Bali SP, Wani FA, Bashir H. An epidemiological study on quality of life among rural elderly population of Northern India. Int J Med Sci Public Health. 2013;2:514-22.

14. Kumar R.Social determinants of health among elderly: An anthropological study. International Journal of Research in Sociology and Social Anthropology. 2013;1(1):11-3.

15. Bharati DR, Pal R, Rekha R,Yamuna TV, Kar S, Radjou AN. Ageing in Puducherry, South India: An overview of morbidity profile. J Pharm Bioallied Sci. 2011;3:537-42.

16. Afridi N, Mahmood H, Mashoori A, Waqar A, Hassan S. Elderly in Abbottabad; Health problems and loneliness. Professional Med J. 2014;21(1):18590.

17. Barman SK, Lata K, Ram R, Ghosh N, Sarker G, Shahnawaz K. A study of morbidity profile of geriatric population in an urban community of Kishanganj, Bihar, India. GJMEDPH 2014;3(1).

18. Shraddha K, Prashantha B, Prakash B. Study on morbidity pattern among elderly in urban population of Mysore, Karnataka, India Int J Med Biomed Res. 2012;1(3):215-23.

19. Kakkar R, Agarwal P, Kandpal SD, Bansal SK. An Epidemiological study to assess morbidity profile among geriatric population in District Dehradun. Indian J of Community Health. 2013;25(1):39-44.

20. Joshi K, Kumar R, Ajith A. Morbidity profile and its relationship with disability and psychological distress among elderly people in northern India. Int $\mathbf{J}$ Epidemiol. 2003;32:978-87.

21. Kumar R, Shafee M. Assessment of morbidity pattern and its correlates among elderly population in rural area of Perambalur, Tamilnadu, India. IJBR. 2014;5(4):247-50.

22. Singh JP, Singh PN, Kariwal P, Singh AK, Kasturwar NK, Srivastava A. Geriatric Life Style Profile in an Urban Slum, Central India. Sch J App Med Sci. 2013;1(4):344-7.

23. Srivastava K, Gupta SC, Kaushal SK, Chaturvedi M. Morbidity profile of elderly. A cross sectional study of Urban Agra. Indian $\mathbf{J}$ of community health. 2009;22(1):51-5.

Cite this article as: Jain S, Kaware AC, Doibale MK, Shaikh S, Radhey BK, Mathurkar MP. Morbidity pattern among geriatric population in urban field practice area of district of Maharashtra: a cross sectional study. Int J Community Med Public Health 2016;3:523-9. 